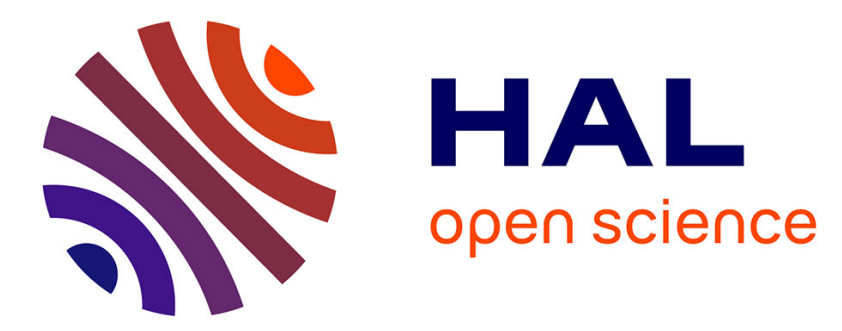

\title{
MULTI-STAINING REGISTRATION OF LARGE HISTOLOGY IMAGES
}

Daniel Felipe Gonzalez Obando, Astri F Frafjord, Inger Øynebråten, Alexandre Corthay, Jean-Christophe F Olivo-Marin, Vannary F Meas-Yedid

\section{- To cite this version:}

Daniel Felipe Gonzalez Obando, Astri F Frafjord, Inger Øynebråten, Alexandre Corthay, JeanChristophe F Olivo-Marin, et al.. MULTI-STAINING REGISTRATION OF LARGE HISTOLOGY IMAGES. 2017 International Symposium on Biomedical Imaging (ISBI 2017), IEEE, May 2017, Melbourne, Australia. hal-01578420

\section{HAL Id: hal-01578420 \\ https://hal.science/hal-01578420}

Submitted on 29 Aug 2017

HAL is a multi-disciplinary open access archive for the deposit and dissemination of scientific research documents, whether they are published or not. The documents may come from teaching and research institutions in France or abroad, or from public or private research centers.
L'archive ouverte pluridisciplinaire HAL, est destinée au dépôt et à la diffusion de documents scientifiques de niveau recherche, publiés ou non, émanant des établissements d'enseignement et de recherche français ou étrangers, des laboratoires publics ou privés.

\section{(1)(1) $\$(0)$}

Distributed under a Creative Commons Attribution - NonCommercial - ShareAlikel 4.0 


\title{
MULTI-STAINING REGISTRATION OF LARGE HISTOLOGY IMAGES
}

\author{
Daniel F González Obando ${ }^{1}$, Astri Frafjord ${ }^{2}$, Inger Øynebråten ${ }^{2}$, Alexandre Corthay ${ }^{2}$, \\ Jean-Christophe Olivo-Marin ${ }^{1}$, Vannary Meas-Yedid ${ }^{1}$ \\ ${ }^{1}$ Institut Pasteur, Bioimage Analysis Unit - CNRS UMR3691, Paris, FRANCE \\ ${ }^{2}$ Oslo University Hospital, Tumor Immunology group, Oslo, NORWAY
}

\begin{abstract}
Quantifying T cells inside tumorous tissue can help identifying immune profiles in order to improve prognosis and possibly develop immunotherapy. However, to identify $\mathrm{T}$ cells and cancerous cells in two consecutive staining slides is challenging: the tissue preparation introduces the problem of alignment on large size images with poor visual common information. This work presents a framework for aligning whole slide images by extracting their common information and performing non-rigid registration based on B-splines to solve this problem. Experiments show good results with a mean error of $20.34 \pm 12.20 \mu \mathrm{m}$ on our images even if some developments are still needed. This preliminary work is publicly available as part of our open-source Icy platform.
\end{abstract}

Index Terms - Histology registration, deformable registration, image analysis, whole slide imaging

\section{INTRODUCTION}

The aim of this project is to align multi-stained histological images in order to characterize and quantify immune cells inside tumorous tissue. The final goal is to develop new prognosis tools and identifying immune profiles associated with good prognosis, which could lead to development of immunotherapy for lung cancer cells. Generally, due to staining limitations within the same slide, multistaining on tissue slides is needed in the pathology field for the diagnosis and prognosis of disease to highlight different structural and/or functional information. The pathologist expert observes multiple slides and then combines the visual information to reach a diagnostic. These serial multi-staining slides provide complementary information but require the image registration step to allow an efficient analysis.

Image registration has been performed to whole slide imaging (WSI) for two mains applications: i) 3D reconstruction of serial sections ii) enable multi-staining and/or multi-modal visualisation and analysis.

Image registration is frequently used on histological images to pair information from different sources (different imaging modalities, different staining techniques, etc.). The principle of image registration lies on the association of common information in two or more images $[1,2,3,4,5,6]$. While the principle of image registration also applies to non-linear registrations, rigid transformations (e.g. cross-correlation based methods [1] or SIFT features [3]) are a specific form of registration. This technique can perform a fast registration, but with an important loss of precision in details, particularly in the tissue local deformations. In contrast to optical sections, the sample preparation protocol (embedding, sectioning, staining, cover-glass application) introduces misalignment and local de- formations between serial sections of tissue. Hence, generally, serial sections can not be successfully registered by a global rigid transform, but need also local deformable transform. In [4], the authors use contrast enhancement along with SIFT and B-spline registration techniques to align histological images. This technique is robust when both images use the same staining protocol (they only need to be color normalized). Nonetheless, when tissue staining differs from image to image this method gives poor results. In [5], a method for 2D and 3D images is introduced using on unsupervised content classification using similarity features based on intensity variation and spatial relationship between classes. Although this method is robust on multi-stained pathological images, a rigid registration based on the original image is performed as first step. This rigid registration on the original intensities might introduce important registration errors when stains are very different, thus affecting the rest of the registration method. These techniques have been successfully tested on regular-sized images, but the registration becomes challenging when dealing with large images as they cannot be fully loaded on memory to be processed and only few works deal with full resolution $[7,6]$.

Histological slides can provide several kinds of information in the same section, but sometimes staining limitations don't allow the desired information in the same slide as can be seen in Fig. 1. These two images share the same geometrical structure but few common elements. To identify tumor cells and T cells, markers were developed using Cytokeratine and CD3 proteins. Unfortunately, T cells cannot be correctly displayed when Cytokeratine is used to show tumor cells. To alleviate this inconvenience, two serial slices of the same tissue are treated with different staining markers. The images are acquired with the AxioScanZ1, at objective 20x, given a spatial resolution of $0.22 \mu \mathrm{m}$ in $\mathrm{x}$ and $\mathrm{y}$.

The challenges for digital pathology are two-fold: i) the dissimilar appearances of the images resulting from the staining, ii) the large image size (typical size: $50 \mathrm{Kx} 50 \mathrm{~K}$ pixels and up to $90 \mathrm{Kx} 90 \mathrm{~K}$ pixels). To address these challenges, we propose to extract the common information and to perform registration on scaled versions of the thresholded images, and then apply the resulting transformation on the full resolution images.

\section{METHOD}

The goal of image registration is to determine a transformation that maximizes the similarity between two images. We propose a registration framework consists of three steps: i) common information extraction, ii) registration combining rigid and deformable registrations and iii) application of the deformation model (Cf. Fig. 2). The first step aims to extract the geometric structure of the marked tissue in both input images by K-Means classification. Then, the model of deformations is computed on these segmented images to 

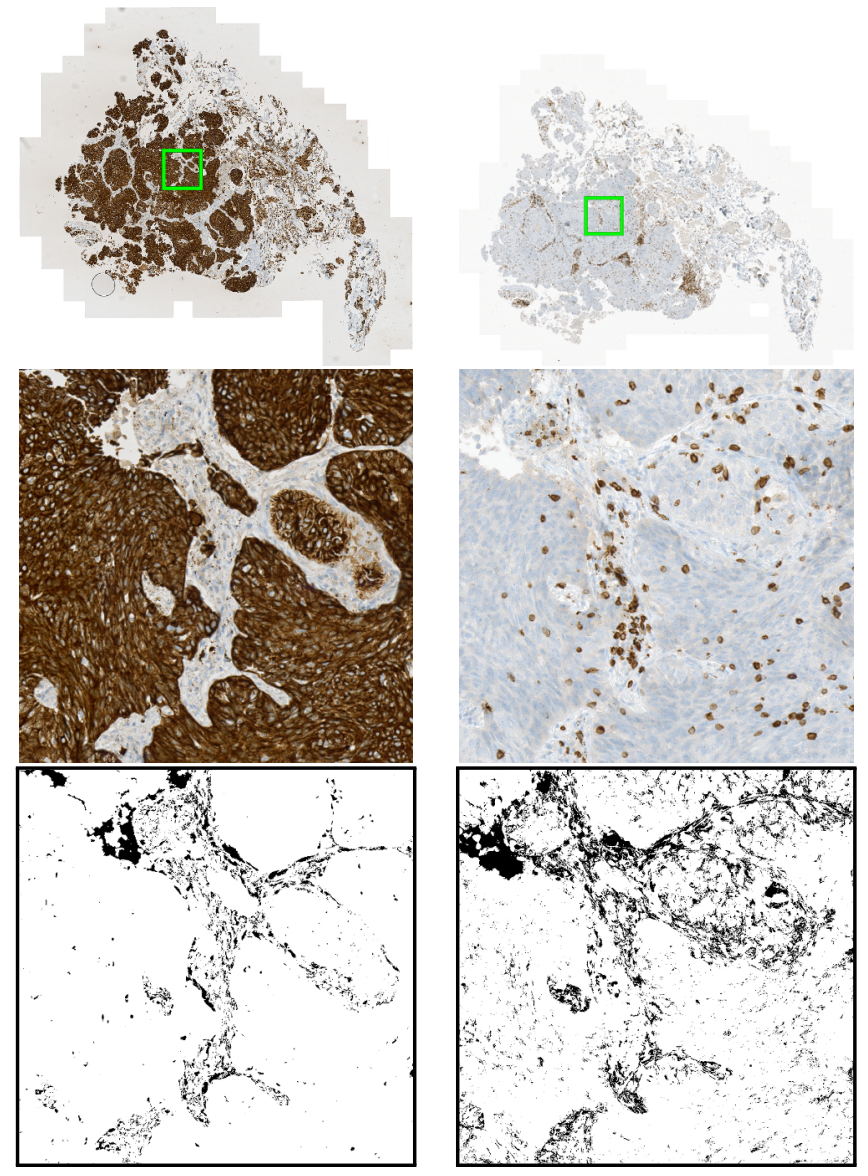

Fig. 1. Example of cancerous lung tissue images. In brown, images are stained with Cytokeratin to highlight cancerous cells (left column), and stained with CD3 to show immune cells (right column). Whole slide images (first row), detail on the samples (second row), and thresholded images to extract common information (third row).

align the tissue structures. Finally, the deformation model is applied to the source image to reconstruct the aligned staining image. The proposed method is based on rigid cross correlation registration followed by an deformable B-spline registration on binary images with common information in source and target images. A pyramidal strategy is used to register decimated input images and the resulting model is applied to build full size registered images.

\subsection{Common information extraction}

Before aligning any image, it is necessary to determine the common information, which will allow for successful alignment. For these images the common information is reduced since the cancerous tissue is not distinguishable in the image of immune cell and the immune cells are not visible in the cancerous tissue sample (Cf. Fig. 1). Instead, the general structure of the sample will be taken into account to perform the registration: the background and stroma (in darker blue) are common in both images. Once this information is segmented, the registration procedure can take place.

Even though both input images contain tissue with a similar shape, a simple registration with the image raw intensities wouldn't work because the registration algorithm uses the image difference as a similarity measure. To solve this problem, it is necessary to pre-process input images to extract their shape. For this, a thresholding is performed using a K-Means classification on the gray-level images histogram to find the threshold value. Different tests were performed in order to find the correct amount of classes and which classes should be used for the thresholding of these images. Test results provided the following information. For the images presenting cancerous cells, using 5 classes to perform the classification and taking the highest class as threshold managed to extract the tissue structure on $100 \%$ of the tested images (8 pairs of WSI); for the images showing $\mathrm{T}$ cells, using 3 classes and taking the highest class as threshold manage to extract the tissue structure on $100 \%$ of the tested images (Cf. third row in Fig. 1). Thanks to these result images, it is then possible to perform a registration, as they make visible in the same way the common information that each image holds.

\subsection{B-spline registration}

This project uses B-splines to model the image deformation to keep the level of detail of the image and to control the deformation level in the registration. Additionally, as B-splines model continuous functions, the transformation model allows to keep the deformation detail when scaling the deformation on large images. The implementation for aligning images is based on the method proposed in [8], which uses intensity variations as registration criteria, soft landmarks, and regularization terms on the splines' divergence and curl. The registration is modeled as an energy minimization functional composed by three terms: data term $E_{i m g}$, landmark term $E_{\mu}$ and regularization term $E_{\text {div }}, E_{\text {rot }}: E=w_{i} E_{i m g}+w_{\mu} E_{\mu}+\left(w_{d} E_{\text {div }}+w_{r} R_{\text {rot }}\right)$. Given the complexity of automatically finding corresponding landmarks on multi-staininig images, the landmark term is not used, thus reduced to 0 .

Using B-Spline coefficients is advantageous for registration because a continuous function can be modeled from a small set of control points holding.

More precisely, images are modeled as surfaces in such way that each point $(x, y)$ of the image has an intensity associated, defined by control points both in $x$ and $y$ directions. Also, this principle is associated to model image transformation, each point in the image has an associated transformation based on control points. In this way, images can support not only affine linear transformations, but also deformable non-linear transformations.

The registration is first performed using a simple cross-correlation registration and then, the non-rigid alignment is done at multiple resolutions in a pyramidal order from coarse to fine details on the image. This can be done thanks to the easy scaling property of Bsplines, which performs a smooth interpolation on the downscaled image. The resulting deformation model is then used to reconstruct the registered full-size image. It is recommended to read [8] to have more details on this registration technique.

\subsection{Handling large images}

To handle large images, full-size images are down-sampled to a manageable scale for the registration method. Then the registration takes place on reduced images and the resulting deformation model is extracted. This model is finally applied to full-size images by upscaling the deformation model (this step keeps the deformation detail thanks to the property of B-splines that provides an accurate interpolation of coordinates and color levels).

Taking advantage of the continuous nature of B-spline surfaces, it is possible to use the registration result of a scaled version of 


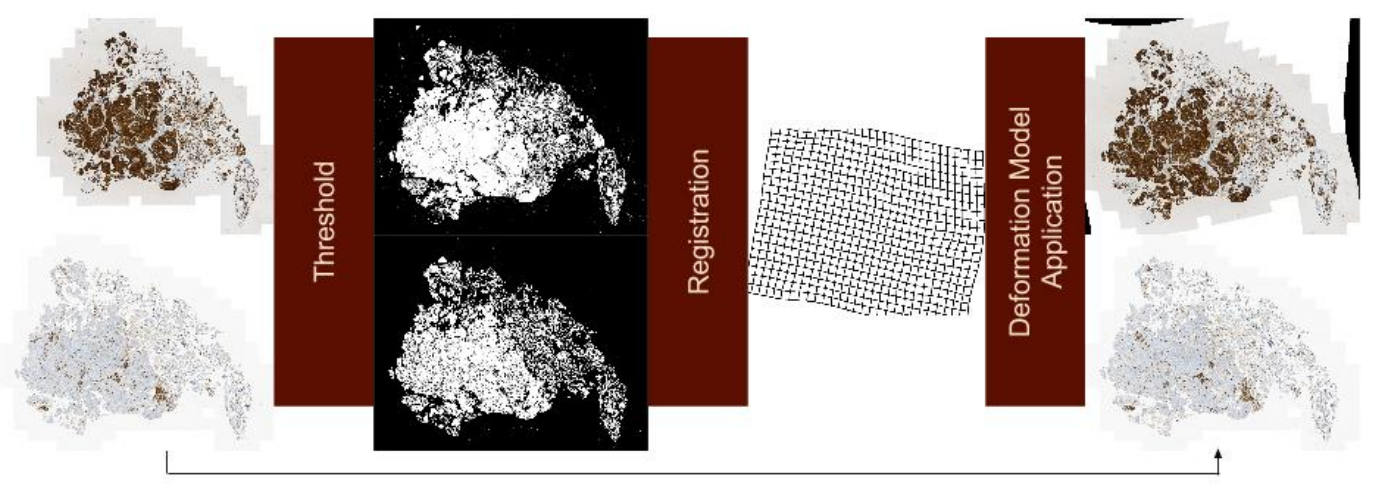

Fig. 2. General framework. From the input source and target images, common information is extracted based on the k-means, followed by computation of the model transformations and finally this deformation model is applied on the source image to obtain the aligned images

the original images and apply it to the full resolution image with high precision, not only on the coordinate correspondences, but also on the interpolation of color intensities of the image. $\mathbf{G}\left(\mathbf{X}_{s}\right)=$ $k \mathbf{g}\left(\mathbf{x}_{s}\right)$, where $\mathbf{G}$ is the transformation model for the full size source image $\mathbf{X}_{s}, k$ is a scaling constant related to the image used for registration $\mathbf{x}_{s}$ and $\mathbf{g}$ is the transformation model computed on the downsampled image.

At this point, it is still not possible to treat the full size image since that would imply to load the whole image and process it to create the registered version and if the image is bigger than the machine RAM, then it won't be possible to create the resulting image. For this problem, a tile processing approach is applied to process the whole image by treating only some image segments at a time, so the memory can handle the process $\left(\mathbf{G}\left(\mathbf{X}_{s}\right)=\left\{\mathbf{G}_{1}\left(\mathbf{X}_{s 1}\right), \mathbf{G}_{2}\left(\mathbf{X}_{s 2}\right), \ldots, \mathbf{G}_{n}\left(\mathbf{X}_{s n}\right)\right\}\right.$, where $\mathbf{G}_{i}$ is the transformation associated to the tile $\left.\mathbf{X}_{s i}\right)$. As the transformation model is computed before creating the resulting full-size image, all tiles will respect the transformation model on tile borders, avoiding the need of stitching tiles. The resulting tile is then stocked back to the hard disk in the registered image file. Finally, the registration result can be found at full resolution in the registered image file.

At the end of the registration process, as both samples are aligned (Cf. Fig. 3), the immune cell quantification on cancerous tissue can be performed.
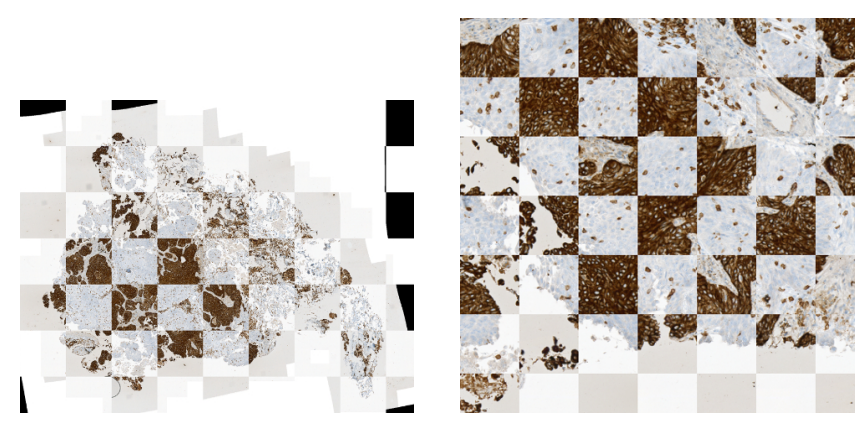

Fig. 3. Aligned images. Chessboard images comparing the result of image alignment performed on a full image (left), and in detail (right) on the cancerous lung tissue.

\section{EXPERIMENTS}

Commonly used evaluation measures include normalized crosscorrelation (NCC) and summed squared difference (SSD) among others. As the original pairs of staining images show poor common information, the NCC could not be used on these images to evaluate the alignment. Instead, in order to evaluate the proposed method, 8 pairs of large images were manually tagged with $N=14$ corresponding control points in both cancerous tissue $\left(p_{s}^{(i)} \mid i \in[1, N]\right)$ and immune cells sample images $\left(p_{t}^{(i)} \mid i \in[1, N]\right)$. The mean distance between corresponding points after registration was used to compute an error measure comparable across different registration methods (Equation 1). This evaluation method was used instead of performing the sum of squared differences between source and target images, because image intensity levels are very different between source and target images. Using SSD would not reflect the accuracy of the registration. Instead, it would only confirm how different these images are [4].

$$
\text { Error }=\frac{1}{N} \sum_{n=1}^{N} D\left(p_{t}^{(n)}, \mathbf{g}\left(p_{s}^{(n)}\right)\right)
$$

The proposed method performance was computed on a machine running Windows 10, with an Intel Core i7-4700MQ Quad-core processor at $2.4 \mathrm{GHz}$ with $8 \mathrm{~GB}$ of RAM. Results showed that full size registration took an average of 70.08 seconds to load images, 6.38 seconds to perform registration, and 1760.62 seconds $(\approx 29$ minutes) to build result images, with an average error of $20.34 \mu \mathrm{m}$ and a standard deviation of $12.20 \mu \mathrm{m}$. We compared our results with a SIFT affine registration, and the average error was $61.86 \mu \mathrm{m}$, with a standard deviation of $28.67 \mu \mathrm{m}$, which means that SIFT is, in average, 3.39 times less accurate than the proposed method. Other methods such as in $[6,5]$ report similar average errors $(\approx 20 \mu \mathrm{m})$. The proposed method was developed using Java, and it is available in the form of a plugin for the Icy bioimage analysis software [9].

\section{DISCUSSION}

The experiment from the previous section, shows the capabilities of registration using B-splines on large biological images with different staining techniques. Aided with different customized parameters, 
users can easily achieve interesting results. Specifically, the low resolution image used to perform the registration showed good results on tested images with a maximum size of $1024 \times 1024$ pixels (scaling tested images by two up to five times). This size was empirically chosen as a good ratio between precision and processing time for this application. To increase precision, it is possible to use a higher size of the low resolution images ( $>1024$ pixels), but of course this incurs on higher processing time.

To date, we have only 8 pairs of whole slide images ( 8 images with cancerous tissue and 8 with T cells) for the validation test. This means that the test should be performed on more images to be really significant but, the tests give good results on all images and can be considered as appropriate for the goal of this project - the registration of these challenging multi-stained images. However, even if the common information extraction provided good results for registration, choosing the correct thresholding parameters using K-Means is still a manual task. This is just a proof of concept, that the registration can be performed correctly on these kind of challenging images. Then, automation on this task is currently a subject of research, by investigating other criteria such as mutual information, texture descriptors, etc., to improve registration accuracy. The implementation can also be improved to take full advantage of multi-threading capabilities of current multi-core processors or of Graphics Processing Units (GPU).

Despite the desirable results obtained by using the proposed method, several assumptions were made in order to assure achievable results. First, the method assumes that both source and target images are contiguous on the block from where they were retrieved (i.e. the have a similar same structure). Allowing to register slides separated with more distance could be interesting. Second, the method assumes that neither tissue folding nor important tissue loss is present in the image. The presence of any of these can increase dissimilarities on the images with the risk of misaligned results.
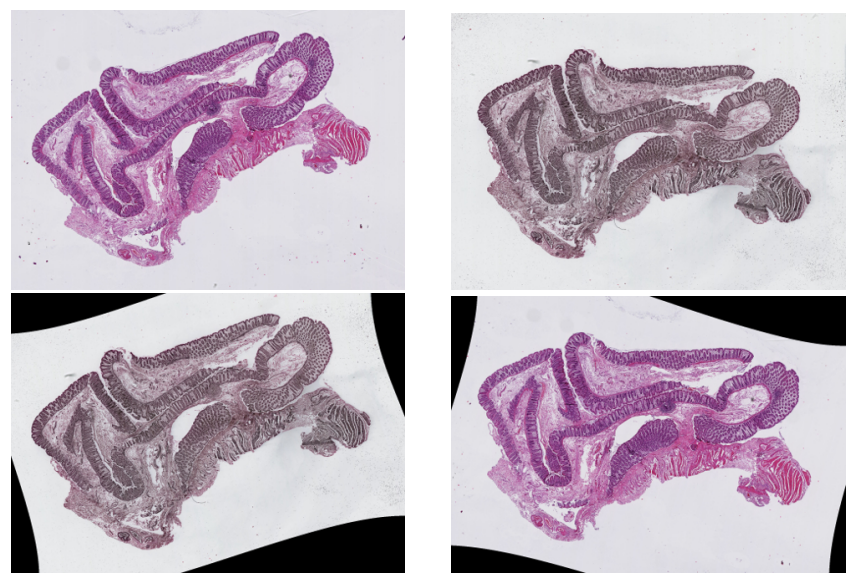

Fig. 4. Test on other staining and tissue. Multi-staining of colon tissue stained with HE (left column) and reticulum staining (right column). Second row, their corresponding registered images

As can be seen in Fig. 4 this approach can perform correctly other staining, but we want also to investigate on multi-modal images. Finally, the current method could be used to perform 3D histological image registration as presented in [5].

\section{CONCLUSION}

We have presented a method to perform large multi-stained histological image registration using B-Splines publicly available ${ }^{1}$. Although the common information extraction has a manual tuning step, obtained results show aligned images suitable for histological analysis. Besides, experiments have shown that good results can be achieved on acceptable execution times. Furthermore, the method implementation is publicly available as part of an open source project. Future work seeks to make common information extraction automatic, as well as improving implementation performance such as parallel computation GPU.

\section{REFERENCES}

[1] Ignacio Arganda-Carreras, Rodrigo Fernandez-Gonzalez, and Carlos Ortiz-de Solorzano, "Automatic registration of serial mammary gland sections," in Engineering in Medicine and Biology Society, 2004. IEMBS'04. 26th Annual International Conference of the IEEE. IEEE, 2004, vol. 1, pp. 1691-1694.

[2] L. Cooper, O. Sertel, J. Kong, G. Lozanski, K Huang, and M. Gurcan, "Feature-based registration of histopathology images with different stains: An application for computerized follicular lymphoma prognosis," Computer Methods and Programs in Biomedicine, vol. 96, no. 3, pp. 182-192, 2009.

[3] Giuseppe Lippolis, Anders Edsjö, Leszek Helczynski, Anders Bjartell, and Niels Chr Overgaard, "Automatic registration of multi-modal microscopy images for integrative analysis of prostate tissue sections," BMC Cancer, vol. 13, no. 1, pp. 1-11, 2013.

[4] Ching-Wei Wang, Shuk-Man Ka, and Ann Chen, "Robust image registration of biological microscopic images," Scientific reports, vol. 4, 2014.

[5] Yi. Song, Darren. Treanor, Andrew. Bulpitt, and Derek. Magee, "3d reconstruction of multiple stained histology images," Journal of Pathology Informatics, vol. 4, no. 2, pp. 7, 2013.

[6] Dan Mueller, Dirk Vossen, and Bas Hulsken, "Real-time deformable registration of multi-modal whole slides for digital pathology," Computerized Medical Imaging and Graphics, vol. 35, pp. 542-556, 2011.

[7] Lee Cooper, Shan Naidu, Gustavo Leone, Joel Saltz, and Kun Huang, "Registering high resolution microscopic images with different histochemical stainings-a tool for mapping gene expression with cellular structures," in Proceedings of the workshop on microscopic image analysis with applications in biomedicine, 2007.

[8] I. Arganda-Carreras, C.Ó.S. Sorzano, P. Thévenaz, A. MuñozBarrutia, J. Kybic, R. Marabini, J.M. Carazo, and C. Ortiz-de Solórzano, "Non-rigid consistent registration of 2D image sequences," Physics in Medicine and Biology, vol. 55, no. 20, pp. 6215-6242, October 2010.

[9] F. de Chaumont, S. Dallongeville, N. Chenouard, N. Herv, S. Pop, T. Provoost, V. Meas-Yedid, Pankajakshan P., T. Lecomte, Y. Le Montagner, T. Lagache, A. Dufour, and J.C. Olivo-Marin, "Icy: an open bioimage informatics platform for extended reproducible research," Nature Methods, vol. 9, no. 7, pp. 690-696, 2012.

\footnotetext{
${ }^{1}$ Available on GitHub: https://github.com/danyfel80/icy-BUnwarp.git as a plugin for Icy
} 